\title{
Origin of Solar System redefined
}

\section{Professor Thomas Gold has stood the conventional view of the origin of the Solar System on its head and seems certain to provoke astrophysicists to a wave of recalculation, even observation.}

Not for the first time, Professor Thomas Gold from Cornell University has provided astrophysicists with a new agenda, this time on the old question of the mechanism by which the Sun and stars like it were formed. Speaking at last week's meeting on rotation in the Solar System, organized in London by the Royal Society, Gold invited his audience to abandon the conventional framework that the Solar System was formed by rapid condensation within a molecular cloud, perhaps in some tens of thousands of years, and that the Sun and planets were then formed by differential condensation within such a compact mass - and to follow instead his own conviction that the condensation was much slower, perhaps extending over several hundreds of millions of years.

Gold's starting point is the now familiar observation that the angular momentum of the Sun is a small part of the angular momentum of the Solar System as a whole -1 part in 180 to be precise. The standard explanation is that during the final stage in the evolution of the Solar System when the planets differentiated from the Sun, angular momentum was transferred to the outer objects from the centre, the rotation of which would otherwise be too rapid for stability. Gold's argument is that the Solar System did not form by the initial collapse and subsequent dispersion of angular momentum (and some material), but that slow accretion onto a central object was dominant from the start.

The chief objective of Gold's argument is to explain why the Sun now embodies such a small proportion of the angular momentum of the Solar System as a whole. On his view but also on the conventional view, the total should of course be that of the portion of the molecular cloud from which the whole Solar System is derived less the amount of angular momentum lost during or after its formation. On both views, the original cloud was larger than the present dimensions of the Solar System, so that its rotational speed would have been less than that of the planets (measured in years) or of the Sun (less than a month).

Gold pointed out last week that if Uranus and Neptune, now largely made of carbon, oxygen and nitrogen, began life as much larger objects with a chemical composition more like that of Jupiter and Saturn, the subsequent loss of large amounts of hydrogen and helium would have entailed a substantial loss of angular momentum, in which case the small fraction of the total now embodied in the Sun would have been even smaller in the past.

What can account for this state of affairs? Gold's explanation is simple but ingenious. Let there be some portion of a molecular cloud which, as a consequence of some density fluctuation, is dense enough to be self-gravitating and thus to have a centre of gravity. If the material is also dense enough for intermolecular collisions to be significant, it will begin to accumulate near the centre until, in the course of time, the centre is occupied by an identifiable mass, a proto-star, by which time at least the inner portion of the cloud will have become disk-like. In this condition, the central object will continue to accrete material from the disk, but with an angular momentum appropriate to the orbital speed at its equator. Plainly such a process of accretion cannot avoid the difficulty that a star grown in this way would have a very large angular momentum unless the growing object were more compact than the Sun. But this, Gold said last week, could be the case if the slowly accreting object were statistically degenerate, perhaps even a neutron star. Then, although the equatorial orbital speed will be very large, the increment of angular momentum will be only small. And the central object will come to resemble a mainsequence star only when accretion makes the central temperature and density of the central object great enough for thermonuclear energy production to begin, whereupon the object will quickly grow in size (oscillating in the process) and, like an ice-skater who stretches out his arms, will then spin more slowly.

But what evidence can there be to guide the choice between Gold's accretion scheme and the conventional view? Each entails the production of the central star from a rotating disk of material. Gold last week drew attention to two supporting lines of evidence - the observation that most non-binary stars are also, like the Sun, slowly rotating objects and that the vectors of the angular momentum of the Sun and of its planetary system point in directions which are more than 7 degrees apart. Gold's argument is that this misalignment is simply not explicable if the planets are the means by which a rotating central object sheds excess angular momentum, but that slow accretion from what might well be a puckered disk can easily accommodate the discrepancy.
It is too soon to know where all this will lead. The ideal would of course be that the same model of slow accretion should also account for the formation of binary stars, which may presumably be the products of the fission of degenerate stars rotating too quickly for stability, but that arithmetic has not yet been done. What is clear is that for single slowly accreting stars, the rate of heat conduction through the degenerate material of the growing but inert proto-star will limit the rate of accretion that can be accommodated without igniting thermonuclear fusion, whence the guess that the formation of visible stars will be slow. An obvious difficulty for the new model, raised last week, is the homogeneity of age of stars in globular clusters and that it may allow too many black holes to form.

Gold himself last week acknowledged that the expansion of a degenerate star would probably not be sufficient to account for the slowness of rotation of the Sun, perhaps by a factor of five, although he argued that mechanisms involving the outward transfer of angular momentum by the agency of magnetic fields (invoked on the conventional view) are more than adequate to bridge this gap. One intriguing possibility is that it may be possible to identify proto-stars in this mould by looking in molecular clouds for Doppler-shifted emission from material travelling with the equatorial orbital speed just above the surface of a rotating degenerate star.

The planetary implications of Gold's model are also potentially fruitful. One possibility is that late in the accretion of the central star, the residue of the molecular cloud might consist of a sequence of rings not all in the same plane; Gold argues that such a system could account not only for the formation of Oort's cometary belt and for the occurrence of chemically inhomogeneous meteoritic material but also for the suggestions that the material of which the Solar System is made has been contaminated with material derived from at least two supernova explosions whose products are not distributed evenly.

Last week, Gold himself was obviously shy of carrying such arguments too far, if only because that would accommodate an ad planetum explanation of each object in the Solar System. Diffidence on that account, natural enough, is not however an argument against Gold's model, which is certain in the next few months (or weeks?) to have people in the field rushing to their computer programs.

John Maddox 